\title{
疏水耐环境型 $\mathrm{SiO}_{2} / \mathrm{TiO}_{2} / \mathrm{SiO}_{2}-\mathrm{TiO}_{2}$ 太阳能宽光谱 增透膜的制备及性能研究
}

\author{
檀满林 ${ }^{1}$, 张礼杰 ${ }^{1,2}$, 王晓伟 ${ }^{1}$ ，马 清 ${ }^{1}$, 符冬菌 ${ }^{1}$, 张维丽 ${ }^{1}$, \\ 李冬霜 ${ }^{1}$, 陈建军 ${ }^{1}$, 张化宇 ${ }^{2}$ \\ (1. 深圳清华大学研究院，深圳 518057; 2. 哈尔滨工业大学深圳研究生院，深圳 518055)
}

摘 要: 利用 TFCcal 设计软件构建膜系结构, 采用溶胶-凝胶工艺和提拉法在超白玻璃上制备出厚度精确可控的宽 光谱、高增透型 $\mathrm{SiO}_{2} / \mathrm{TiO}_{2} / \mathrm{SiO}_{2}-\mathrm{TiO}_{2}$ 减反膜，同时结合甲基三乙氧基硅烷(MTES)改性碱催化的 $\mathrm{SiO}_{2}$ 溶胶，通过提 拉法一次制备出高透过率疏水型薄膜。研究表明, 高增透型三层宽光谱减反膜的理论膜层厚度依次为: $80.9 \mathrm{~nm}$ (内 层 $\mathrm{SiO}_{2}-\mathrm{TiO}_{2}$ )、125.0 $\mathrm{nm}$ (中间层 $\mathrm{TiO}_{2}$ )、95.5 nm(外层 $\mathrm{SiO}_{2}$ ), 其在 400 700 nm 可见光范围内平均透过率实际可高 达 $97.03 \%$ 以上。多层膜经过退火处理后, 膜面的水接触角高达 $131.5^{\circ}$, 同时陈化两个月以后的多层膜透过率仅下降 $0.143 \%$ ，表明制备的 $\mathrm{SiO}_{2} / \mathrm{TiO}_{2} / \mathrm{SiO}_{2}-\mathrm{TiO}_{2}$ 多层减反膜具有优良的疏水和耐环境性能。

关 键 词: 增透膜; 疏水型; 耐环境; 宽光谱

中图分类号: TB321 文献标识码: A

\section{Hydrophobic and Environment-resistant Properties of $\mathrm{SiO}_{2} / \mathrm{TiO}_{2} / \mathrm{SiO}_{2}-\mathrm{TiO}_{2} \mathrm{Mul}-$ tilayer Antireflective Films in Wide Solar Spectra Range}

TAN Man-Lin ${ }^{1}$, ZHANG Li-Jie ${ }^{1,2}$, WANG Xiao-Wei ${ }^{1}$, MA Qing ${ }^{1}$, FU Dong-Ju ${ }^{1}$, ZHANG Wei-Li ${ }^{1}$, LI Dong-Shuang ${ }^{1}$, CHEN Jian-Jun ${ }^{1}$, ZHANG Hua-Yu ${ }^{2}$

(1. Research Institute of Tsinghua University in Shenzhen, Shenzhen 518057, China; 2. Harbin Institute of Technology Shenzhen Graduate School, Shenzhen 518055, China)

\begin{abstract}
In the help of coatings optimization design using TFCcal program, wide spectrum antireflective $\mathrm{SiO}_{2} /$ $\mathrm{TiO}_{2} / \mathrm{SiO}_{2}-\mathrm{TiO}_{2}$ multilayer films with thickness precisely controlled were prepared on low iron glass using sol gel process and Czochralski method. The thickness of above layers were separately set as $80.9 \mathrm{~nm}\left(\mathrm{SiO}_{2}-\mathrm{TiO}_{2}\right.$, inner layer), $125.0 \mathrm{~nm}\left(\mathrm{TiO}_{2}\right.$, inter-layer) and $95.5 \mathrm{~nm}\left(\mathrm{SiO}_{2}\right.$, outer layer) according to the results of calculation. A high transmission and highly hydrophobic film was prepared by Czochralski method combined with methyl triethoxysilane (MTES) $\mathrm{SiO}_{2}$ modified base-catalyzed sol. The average optical transmittance could reach $97.03 \%$ in the wavelength range of $400-700 \mathrm{~nm}$. After annealing treatment, the surface water contact angles were almost around $131.5^{\circ}$. Furthermore, the optical transmittance only reduced $0.143 \%$ after aging for two months, showing that the prepared $\mathrm{SiO}_{2} / \mathrm{TiO}_{2} / \mathrm{SiO}_{2}-\mathrm{TiO}_{2}$ multilayer antireflective films had excellent hydrophobic and environment resistant properties.
\end{abstract}

Key words: antireflective films; hydrophobic; environment resistant; wide spectrum 
减反射(增透)膜能够有效地降低光的反射率而 被广泛应用于建筑玻璃、光伏电池板、太阳能集热 管和高功率激光器等领域 ${ }^{[1-5]}$ 。用于太阳能电池组件 表面的封装玻璃，其上下表面的反射率可高达 $8 \%$ 左右。如果在玻璃表面镀上一层减反射膜, 将对增 加光子的入射量, 提高电池组件的光电转换效率具 有重要意义。溶胶-凝胶法以其工艺操作简单、成本 低廉而被广泛应用于制备太阳能减反射膜 ${ }^{[6-7]}$ 。普通 的单层增透膜主要采用碱催化正硅酸乙酯(TEOS) 制备的 $\mathrm{SiO}_{2}$ 溶胶作为前驱体, 由溶胶中 $\mathrm{SiO}_{2}$ 小颗粒 在基底表面堆积而成，因此薄膜的孔隙率大、折射 率低，在单一波长上透过率高达 $100 \%$ 。但是单层增 透膜的增透波段范围非常小, 只是在某一特定波长 处透过率高, 其他波段透过率都非常低且耐摩擦性 能差，导致其使用年限较短，限制了实际的应用 ${ }^{[8]}$ 。 酸/碱复合催化法能够使制备的 $\mathrm{SiO}_{2}$ 增透膜的耐摩 擦性能得到显著提高, 但其在可见光区域增透范围 仍然较小，且极易吸收周围环境的水分及悬浮颗粒 而导致光学性能迅速下降 ${ }^{[9]}$ 。鉴于减反射膜的耐环 境性能差, 人们分别以酸催化的 $\mathrm{SiO}_{2}$ 和 $\mathrm{TiO}_{2}$ 作为 内外层, 制备了 $\mathrm{SiO}_{2} / \mathrm{TiO}_{2}$ 双层减反射膜。外层 $\mathrm{TiO}_{2}$ 的光催化效应使得薄膜能够分解周围环境的有机物 而达到自清洁功效, 但是其较高的折射率导致双层减 反膜在可见光范围内平均透过率只有 $95 \%$ 左右 ${ }^{[10]}$ 。双 层 $\mathrm{W}$ 型的 $\lambda / 4-\lambda / 2$ 增透膜采用酸催化 $\mathrm{SiO}_{2}$ 膜作为膜 系外层, $\mathrm{TiO}_{2}$ 薄膜作为膜系内层, 提高了薄膜的透 过率 ${ }^{[6]}$, 但这种双层增透膜也只能在一个或者两个 波长处达到最大透过率, 而不能在整个可见光光谱 区间内具有高增透性能。考虑到增透膜增透区间范 围窄, 人们设计了 $\mathrm{SiO}_{2}-\mathrm{TiO}_{2}$ 多层减反射膜, 薄膜在 400 800 nm 范围平均透过率非常高, 但薄膜耐环境 性能差 ${ }^{[11]}$ 。

为了使制备的减反膜具备高透过率兼高疏水性 和而环境性能, 本实验通过 TFCcal膜系设计软件设 计出 $\mathrm{SiO}_{2} / \mathrm{TiO}_{2} / \mathrm{SiO}_{2}-\mathrm{TiO}_{2}$ 三层膜层结构, 通过采用 甲基三乙氧基硅烷改性碱催化法引入疏水基团作为 外层 $\mathrm{SiO}_{2}$ 层, 以期获得高质量的太阳能宽光谱增透 膜, 实现其在光伏电池组件和太阳能平板集热器领 域的广泛应用。

\section{1 膜系设计}

目前光学薄膜的设计方法种类多, 包括适量作 图法、解析合成法和导纳圆图法等方法, 其中计算 机辅助设计法具备快速直观等特点 ${ }^{[12]}$ 。本设计采用
$\mathrm{TFCcal}$ 软件优化三层膜的厚度及折射, 膜系结构为 $\lambda / 4-\lambda / 2-\lambda / 4$, 各层折射率从里层到外层依次为 1.7 、 $2.2 、 1.44$, 膜层厚度从里到外依次为 80.9、125.0、 $95.5 \mathrm{~nm}$ 。考虑到 $\mathrm{SiO}_{2}$ 和 $\mathrm{TiO}_{2}$ 薄膜在可见光范围内 具备良好的消光性能，且两者的折射率可通过控制 热处理温度达到模拟所需的 1.44 和 2.2 , 复合层折 射率为 1.7 可通过向 $\mathrm{SiO}_{2}$ 溶胶中掺杂不同比例的 $\mathrm{TiO}_{2}$ 溶胶来获得, 本设计的三层膜采用的材料选定 为 $\mathrm{SiO}_{2}$ 和 $\mathrm{TiO}_{2}$, 膜层理论结构参数见表 1 , 膜系设 计出的理论反射曲线如图 1 所示。

\section{2 实验方法}

\section{1 准备样品}

1) 酸催化 $\mathrm{TiO}_{2}$ 溶胶 ${ }^{[1]}$

将钛酸四丁酯、无水乙醇、去离子水和浓盐酸按 照摩尔比为 $1: 49.75: 3.55: 0.22$ 的比例混合, 在常温下 反应 $2 \mathrm{~h}$ 得 $\mathrm{TiO}_{2}$ 溶胶, 再把 $\mathrm{TiO}_{2}$ 溶胶放入 $30^{\circ} \mathrm{C}$ 的恒 温箱中陈化备用。

2) 酸催化 $\mathrm{SiO}_{2}$ 溶胶 ${ }^{[11]}$

将正硅酸乙酯、无水乙醇、去离子水和浓盐酸 按照摩尔比为 $1: 36.83: 4.01: 4.16 \times 10^{-3}$ 的比例混合, 在常温下反应 $2 \mathrm{~h}$ 后得 $\mathrm{SiO}_{2}$ 溶胶。

表 1 膜层理论结构参数

Table 1 Calculated parameters of optimized multilayer antireflective film

\begin{tabular}{ccc}
\hline \multicolumn{2}{c}{ Refractive index } & Layer thickness $/ \mathrm{nm}$ \\
\hline Glass & 1.52 & - \\
$\mathrm{SiO}_{2}-\mathrm{TiO}_{2}$ & 1.71 & 80.5 \\
$\mathrm{TiO}_{2}$ & 2.20 & 125.0 \\
$\mathrm{SiO}_{2}$ & 1.44 & 95.5 \\
\hline
\end{tabular}

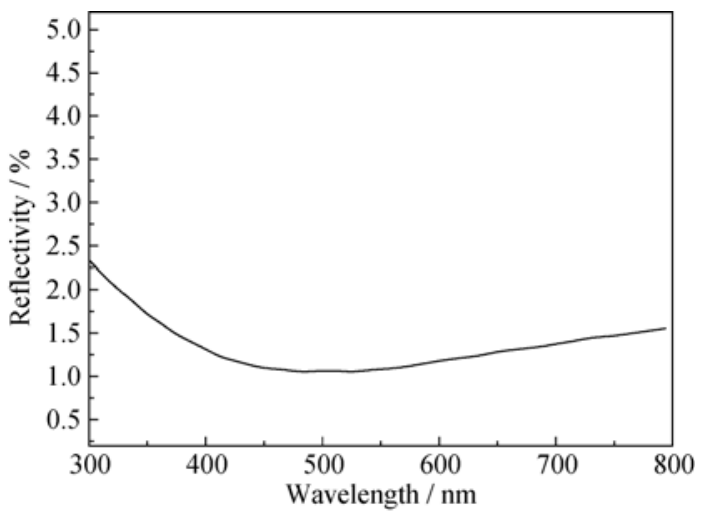

图 1 三层减反射膜理论反射率曲线

Fig. 1 Calculated reflectivity curve of triple-layer antireflective film 


\section{3) $\mathrm{SiO}_{2}-\mathrm{TiO}_{2}$ 复合溶胶}

把上述制备好的 $\mathrm{TiO}_{2}$ 和 $\mathrm{SiO}_{2}$ 溶胶按照一定的 质量百分比混合反应 $2 \mathrm{~h}$, 得 $\mathrm{SiO}_{2}-\mathrm{TiO}_{2}$ 复合溶胶, 取出放入 $30^{\circ} \mathrm{C}$ 的恒温箱中陈化 $7 \mathrm{~d}$ 后, 用 $0.22 \mu \mathrm{m}$ 滤膜过滤备用。

\section{4) 碱催化 $\mathrm{SiO}_{2}$ 溶胶}

分别将正硅酸乙酯、无水乙醇、去离子水按照 摩尔比为 $1: 80: 3$ 的比例放入烧杯中, 在常温下反应 $2 \mathrm{~h}$, 用浓氨水调节 $\mathrm{pH}$ 到 8 左右, 陈化 $6 \mathrm{~d}$ 后在 $80^{\circ} \mathrm{C}$ 下回流 $16 \mathrm{~h}$ 除去氨气, 用 $0.22 \mu \mathrm{m}$ 的有机系聚偏氟 乙烯滤膜过滤备用。

\section{5) 疏水溶胶}

以甲基三乙氧基硅烷(MTES)为前驱体, 以无水 乙醇为互溶剂, 浓盐酸为催化剂, 在常温下将 MTES、无水乙醇、去离子水、浓盐酸按照摩尔比 为 $1: 40: 1: 0.01$ 的比例混合, 剧烈搅拌 $2 \mathrm{~h}$ 后, 陈化 $6 \sim 9 \mathrm{~d}$ 得到疏水溶胶。

\section{6) 疏水改性 $\mathrm{SiO}_{2}$ 溶胶}

把陈化好的各组碱催化 $\mathrm{SiO}_{2}$ 溶胶按不同的体积 比与疏水溶胶混合反应 $30 \mathrm{~min}$ 后, 常温下陈化 2 3 d, 用 $0.22 \mu \mathrm{m}$ 有机系聚偏氟乙烯滤膜过滤备用。

\section{2 制备减反膜}

首先, 在经洗涤剂、丙酮、无水乙醇、去离子 水各超声清洗 $15 \mathrm{~min}$ 后经氮气吹干的超白玻璃上 制备得到的 $\mathrm{SiO}_{2}-\mathrm{TiO}_{2}$ 复合溶胶, $\mathrm{TiO}_{2}$ 溶胶和疏水 改性 $\mathrm{SiO}_{2}$ 溶胶通过浸渍提拉法以一定提拉速度先 镀上 $\mathrm{SiO}_{2}-\mathrm{TiO}_{2}$ 膜, $80^{\circ} \mathrm{C}$ 下干燥 $10 \mathrm{~min}$, 再在 $400^{\circ} \mathrm{C}$ 下轱烧 $2 \mathrm{~h}$ 作为第一层; 然后, 在上述的 $\mathrm{SiO}_{2}-\mathrm{TiO}_{2}$ 膜上镀 $\mathrm{TiO}_{2}$ 膜, 在 $400{ }^{\circ} \mathrm{C}$ 下热处理 $2 \mathrm{~h}$ 后完成第二 层膜制备; 最后, 在制备的 $\mathrm{SiO}_{2}-\mathrm{TiO}_{2} / \mathrm{TiO}_{2}$ 双层膜 基础上, 利用制备的疏水 $\mathrm{SiO}_{2}$ 溶胶镀制第三层(最外 层)疏水改性 $\mathrm{SiO}_{2}$ 层。制备的 $\mathrm{SiO}_{2} / \mathrm{TiO}_{2} / \mathrm{SiO}_{2}-\mathrm{TiO}_{2}$ 三层复合膜在 $200^{\circ} \mathrm{C}$ 热处理 $30 \mathrm{~min}$, 再在 $400^{\circ} \mathrm{C}$ 下 热处理 $30 \mathrm{~min}$, 得到 $\mathrm{SiO}_{2} / \mathrm{TiO}_{2} / \mathrm{SiO}_{2}-\mathrm{TiO}_{2}$ 疏水性减 反膜。

\section{3 测试表征}

采用 M-2000UI 型(J.A.Woollan Co)椭偏仪对各 膜层厚度和折射率进行拟合, 通过拟合所得到的厚 度和折射率参数来修改实验参数。利用日本岛津生 产的 UV2600 测量实验中各组薄膜的透过率, 不同 工艺参数下制备的薄膜经热处理后, 以空气为背景 扫基线, 测量样品的透射率变化规律。采用扫描电 子显微镜(Tescan MIRA3 XMH)观察外层薄膜的断 面形貌并判定膜层厚度。采用视频光学接触角测量 仪(Dataphysics DCAT20)测量薄膜与水的接触角。

\section{3 结果与讨论}

\section{1 $\mathrm{TiO}_{2}$ 含量对 $\mathrm{TiO}_{2}-\mathrm{SiO}_{2}$ 膜折射率的影响}

$\mathrm{TiO}_{2}$ 为高折射率材料, $\mathrm{SiO}_{2}$ 为低折射率材料, 向 $\mathrm{SiO}_{2}$ 溶胶中掺杂不同量的 $\mathrm{TiO}_{2}$ 溶胶可以调节 $\mathrm{TiO}_{2}-\mathrm{SiO}_{2}$ 薄膜的折射率, 使其与膜系设计软件设计 的理论值匹配。实验结果表明, 随着 $\mathrm{TiO}_{2}$ 含量的增 加, $\mathrm{TiO}_{2}-\mathrm{SiO}_{2}$ 膜的折射率不断增大, 当 $\mathrm{TiO}_{2}$ 含量在 $20 \mathrm{~mol} \% \sim 70 \mathrm{~mol} \%$ 之间时, $\mathrm{TiO}_{2}-\mathrm{SiO}_{2}$ 薄膜的折射率呈 线性增长关系, 实验结果如图 2 所示。

\section{2 薄膜透过率}

对于 $\mathrm{SiO}_{2} / \mathrm{TiO}_{2} / \mathrm{SiO}_{2}-\mathrm{TiO}_{2}$ 复合膜, 各层薄膜的厚 度和折射率匹配问题是影响薄膜最终透过率的关键 因素, 薄膜的厚度可以通过改变提拉速度来控制。

\subsection{1 $\mathrm{TiO}_{2}$ 层膜厚对三层膜透过率的影响}

在 $\mathrm{SiO}_{2} / \mathrm{TiO}_{2} / \mathrm{SiO}_{2}-\mathrm{TiO}_{2}$ 膜的制备过程中为了保证 单一变量关系, 在保证第一层 $\mathrm{SiO}_{2}-\mathrm{TiO}_{2}$ 层和最外层 $\mathrm{SiO}_{2}$ 疏水层的厚度不变且都为膜系设计软件所要求 值的前提下, 通过改变提拉速度来调节 $\mathrm{TiO}_{2}$ 层的厚 度。采用分光光度计测得三层膜的透射率曲线如图 3 所示, 从图中可知, 在 350 450 nm 区间段各组 $\mathrm{SiO}_{2} /$ $\mathrm{TiO}_{2} / \mathrm{SiO}_{2}-\mathrm{TiO}_{2}$ 薄膜平均透过率都高达 $98 \%$; 随着 $\mathrm{TiO}_{2}$ 层厚度的增加，在 450 700 nm 区间段，三层膜的 透过率大小依次为 $\mathrm{c}>\mathrm{b}>\mathrm{a}>\mathrm{d}>\mathrm{e}>\mathrm{f}$, 即随着 $\mathrm{TiO}_{2}$ 厚 度的增加, $\mathrm{SiO}_{2} / \mathrm{TiO}_{2} / \mathrm{SiO}_{2}-\mathrm{TiO}_{2}$ 薄膜透过率先增加后 减小, 当提拉速度为 400 和 $420 \mathrm{~mm} / \mathrm{min}$ 时, 中间 $\mathrm{TiO}_{2}$ 层膜厚分别为 $121 \mathrm{~nm}$ 和 $127 \mathrm{~nm}$, 非常接近理论要 求厚度 $125.0 \mathrm{~nm}$ 时, 三层膜的透过率最高。其他各 组 $\mathrm{TiO}_{2}$ 层, $\mathrm{a}$ 组 $\mathrm{TiO}_{2}$ 膜厚小于理论值, $\mathrm{d} 、 \mathrm{e} 、 \mathrm{f}$ 三组 $\mathrm{TiO}_{2}$ 膜厚高于理论值, 且 $\mathrm{TiO}_{2}$ 层厚度偏离理论

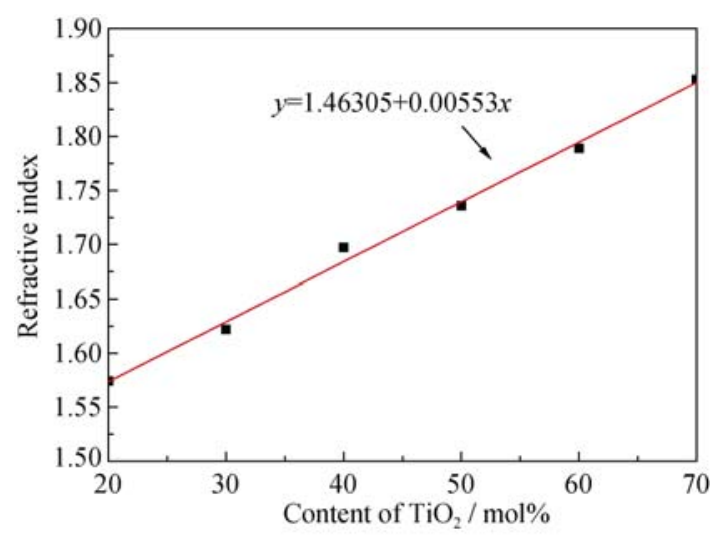

图 $2 \mathrm{TiO}_{2}$ 含量对 $\mathrm{TiO}_{2}-\mathrm{SiO}_{2}$ 薄膜折射率的影响

Fig. 2 Effect of $\mathrm{TiO}_{2}$ content on the refractive index of $\mathrm{TiO}_{2}-\mathrm{SiO}_{2}$ film 


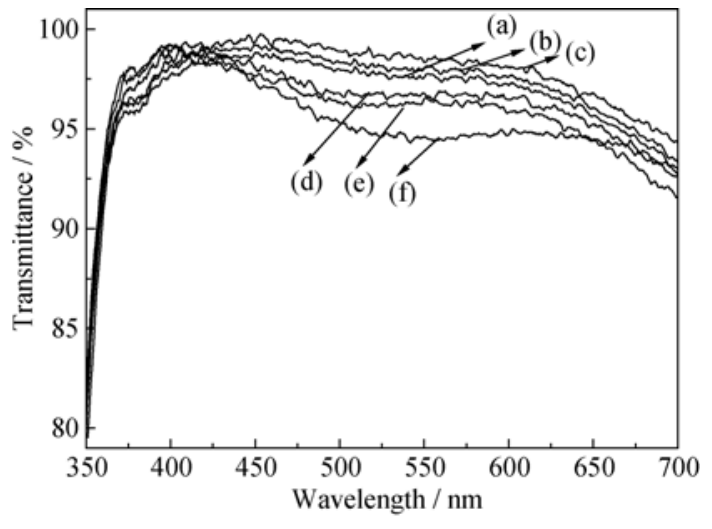

图 $3 \mathrm{TiO}_{2}$ 层不同提拉速度制备的三层膜透过率

Fig. 3 Transmittance of triple-layer antireflective film with $\mathrm{TiO}_{2}$ layer prepared at different pulling rates

(a) $380 \mathrm{~mm} / \mathrm{min}$; (b) $400 \mathrm{~mm} / \mathrm{min}$; (c) $420 \mathrm{~mm} / \mathrm{min}$; (d) $440 \mathrm{~mm} / \mathrm{min}$; (e) $460 \mathrm{~mm} / \mathrm{min}$; (f) $480 \mathrm{~mm} / \mathrm{min}$

值越大, 薄膜高透过率区间范围越窄。由上述结果 可知, 中间 $\mathrm{TiO}_{2}$ 层厚度决定了三层膜的高透过率区 间范围的大小, 任何偏离 $\mathrm{TiO}_{2}$ 理论厚度值都会造成 整个增透区间的减小, 也就是说半波长 $\mathrm{TiO}_{2}$ 层, 起 到了平滑过渡透过率曲线作用。

\subsection{2 $\mathrm{SiO}_{2}$ 层膜厚对三层膜透过率的影响}

保证 $\mathrm{SiO}_{2}-\mathrm{TiO}_{2}$ 最里层和中间 $\mathrm{TiO}_{2}$ 层厚度不变 的情况下, 通过改变提拉速度来调节疏水 $\mathrm{SiO}_{2}$ 层的 厚度。采用分光光度计测得三层膜的透过率曲线如 图 4 所示, 从图中可以看出, 膜层在 375 450 $\mathrm{nm}$ 波 长范围, 保持着高的透过率, 而长波段区间三层膜 透过率逐渐减小, 但是 450 700 $\mathrm{nm}$ 区间段, 薄膜透 过率变化不大, 这也说明中间 $\mathrm{TiO}_{2}$ 层的平滑过渡透 过率曲线的作用。随着提拉速度的增大, 各组薄膜的 透过率大小顺序为 $\mathrm{d}>\mathrm{c}>\mathrm{e}>\mathrm{b}>\mathrm{a}$, 即随提拉速度的 增加, $\mathrm{SiO}_{2} / \mathrm{TiO}_{2} / \mathrm{SiO}_{2}-\mathrm{TiO}_{2}$ 薄膜整个可见光范围内

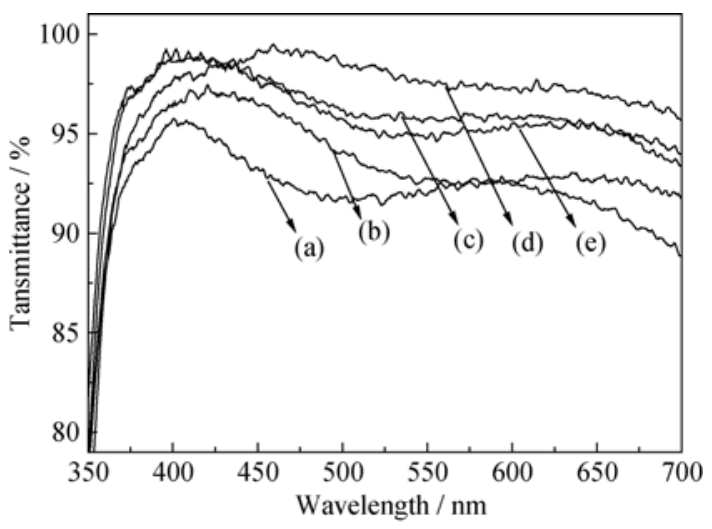

图 $4 \mathrm{SiO}_{2}$ 层不同提拉速度制备的三层膜的透过率

Fig. 4 Transmittances of triple-layer antireflective films with $\mathrm{SiO}_{2}$ layer prepared at different pulling rates

(a) $380 \mathrm{~mm} / \mathrm{min}$; (b) $400 \mathrm{~mm} / \mathrm{min}$; (c) $420 \mathrm{~mm} / \mathrm{min}$; (d) $440 \mathrm{~mm} / \mathrm{min}$; (e) $460 \mathrm{~mm} / \mathrm{min}$; (f) $480 \mathrm{~mm} / \mathrm{min}$
透过率先增大后减小，当提拉速度为 $190 \mathrm{~mm} / \mathrm{min}$ 时, $\mathrm{SiO}_{2}$ 层厚度为 $98 \mathrm{~nm}$, 接近膜系设计理论值; 而 $a 、 b 、 c$ 三组 $\mathrm{SiO}_{2}$ 层厚度小于理论值, $\mathrm{e}$ 组 $\mathrm{SiO}_{2}$ 层厚 度大于理论值。总之, 在保证前两层厚度达到理论 值的前提下, $\mathrm{SiO}_{2}$ 厚度决定了膜层最终平均透过率 大小。

\subsection{3 工艺参数优化后的薄膜透过率}

综合提拉速度对膜厚的影响以及膜厚对薄膜透 过率影响, 制备三层膜的最佳工艺参数, 包括各层 膜的提拉速度、膜厚以及膜层折射率见表 2 所示。 实验中疏水 $\mathrm{SiO}_{2}$ 层提拉速度为 $190 \mathrm{~mm} / \mathrm{min}$ ，中间 $\mathrm{TiO}_{2}$ 层提拉速度为 $420 \mathrm{~mm} / \mathrm{min}$, 内层 $\mathrm{SiO}_{2}-\mathrm{TiO}_{2}$ 复 合层提拉速度为 $190 \mathrm{~mm} / \mathrm{min}$ 时, 薄膜的最终透过 率如图 5 所示, 从图中可以看出三层薄膜在 400 $700 \mathrm{~nm}$ 波长范围，平均透过率达到 $97.03 \%$ 。

图 6 为薄膜断面 SEM 照片, 可以看出各膜层厚 度除最里层 $\mathrm{SiO}_{2}-\mathrm{TiO}_{2}$ 层厚度偏离理论厚度值较大 外，中间层和最外层均非常接近理论膜厚值，制备 的薄膜透过率较高。薄膜透过率比膜系设计理论值 稍微偏低, 这主要是由于提拉过程中各层膜厚与理 论膜存在一定的偏差以及在理论设计时薄膜折射率

\begin{tabular}{|c|c|c|c|c|c|}
\hline & \multicolumn{2}{|c|}{ Calculated } & \multicolumn{3}{|c|}{ Experimental } \\
\hline & $\begin{array}{l}\text { Refractive } \\
\text { index }\end{array}$ & $\begin{array}{c}\text { Layer } \\
\text { thick- } \\
\text { ness/nm }\end{array}$ & $\begin{array}{c}\text { Refractive } \\
\text { index }\end{array}$ & $\begin{array}{c}\text { Layer } \\
\text { thick- } \\
\text { ness/nm }\end{array}$ & $\begin{array}{c}\text { Pulling } \\
\text { rate/ } \\
\left(\mathrm{nm} \cdot \mathrm{min}^{-1}\right)\end{array}$ \\
\hline Glass & 1.52 & - & 1.52 & - & - \\
\hline $\mathrm{SiO}_{2}-\mathrm{TiO}_{2}$ & 1.70 & 80.9 & 1.70 & 72 & 190 \\
\hline $\mathrm{TiO}_{2}$ & 2.20 & 125.0 & 2.20 & 127 & 420 \\
\hline $\mathrm{SiO}_{2}$ & 1.44 & 95.5 & 1.44 & 96 & 190 \\
\hline
\end{tabular}

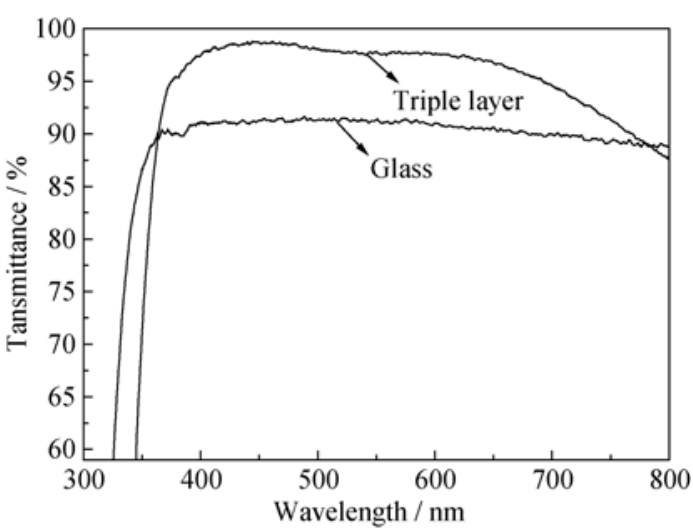

图 5 最佳工艺参数下三层膜透过率曲线

Fig. 5 Transmittance curve of triple-layer antireflective film with optimized process parameters 


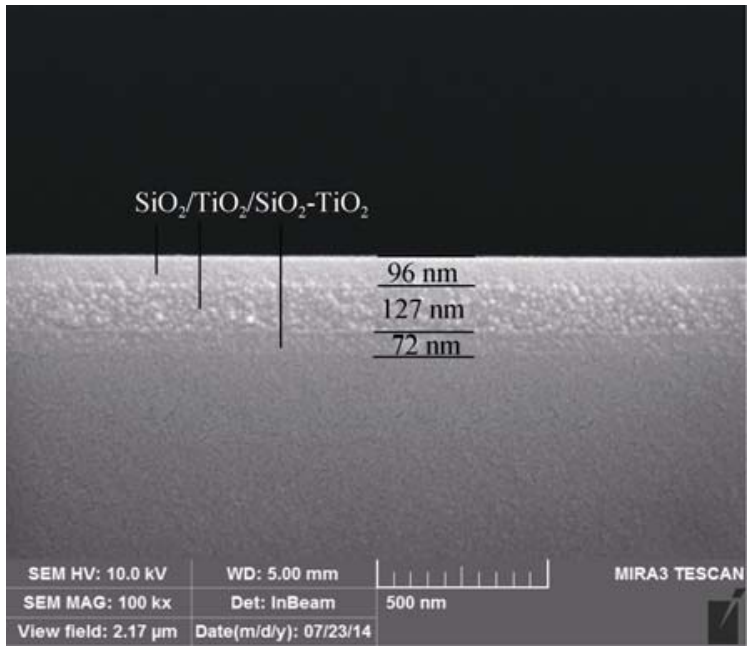

图 $6 \mathrm{SiO}_{2} / \mathrm{TiO}_{2} / \mathrm{SiO}_{2}-\mathrm{TiO}_{2}$ 多层膜截面 $\mathrm{SEM}$ 照片

Fig. 6 Sectional image of $\mathrm{SiO}_{2} / \mathrm{TiO}_{2} / \mathrm{SiO}_{2}-\mathrm{TiO}_{2}$ multiplayer antireflective film

都是以某一特定波长处的折射率为参考值, 忽略了 实际中材料折射率随波长变化而发生改变的情况。

\section{3 疏水性能}

$\mathrm{SiO}_{2} / \mathrm{TiO}_{2} / \mathrm{SiO}_{2}-\mathrm{TiO}_{2}$ 三层膜结构中, $\mathrm{SiO}_{2}$ 层作为 最外层, 由于膜表面富含亲水基团 $(\mathrm{Si}-\mathrm{OH})$, 易吸收 周围环境的水汽, 导致三层膜的透光率下降。此外空 气中的粉尘也易吸附于薄膜表层, 如果薄膜不具备自 清洁性能将会对薄膜透过率造成影响。针对减反射膜 吸潮引起薄膜透过率下降问题可从以下三个方面考 虑: (1)通过改变薄膜表面粗糙度来获得疏水性 ${ }^{[13]}$, 其 原理同荷叶疏水原理一样。此方法虽然可在一定程度 上提高薄膜的疏水性, 但是只能利用增加漫反射来减 少反射率, 未能从根本上解决减反射; (2)对膜层进行 后处理工艺, 通过在疏水性试剂中浸泡后再热处理或 在一定气氛条件下热处理等方式, 引入疏水基团来取 代薄膜表面部分 $\mathrm{Si}-\mathrm{OH}$ 等亲水基团, 从而提高减反射 膜的疏水性 ${ }^{[14]}$, 该方法缺点是膜处理时间长, 效率低 下, 不易推广; (3)对膜层进行修饰改性使其具备疏水 性 ${ }^{[15]}$, 即通过掺杂等方式, 用惰性有机基团置换或部 分置换具备活性的-OH 基团。通常采用疏水性的烷氧 基团来代替亲水性- $\mathrm{OH}$ ，在防水的同时也防灰尘污染， 应用价值高。碱催化制备的 $\mathrm{SiO}_{2}$ 溶胶镀膜后孔隙率大, 膜层折射率偏低且膜层耐摩擦性能欠佳, 为了进一步 提高薄膜折射率和膜层附着力, 本实验通过调节醇硅 摩尔比和添加甲基三乙氧基硅烷溶胶来减小颗粒粒 径和膜层孔隙率，同时掺杂甲基三乙氧基硅烷溶胶来 增加膜层附着力和疏水性能。

\subsubsection{MTES 掺杂浓度对薄膜疏水性能的影响}

采用微量移液器将水滴轻轻滴加在有碱催化
$\mathrm{SiO}_{2}$ 薄膜表面, 发现未掺杂的薄膜表面水滴完全舒展 开来, 表现出非常好的亲水性。随着 MTES 掺杂比例 的增加, 水滴在薄膜表面逐渐突起来, 并逐渐转变为 半球形甚至变成球形, 薄膜与水接触角大小如图 7 所 示。从图 7 可以看出掺杂 MTES 比例为 $0 \sim 50 \mathrm{~mol} \%$ 的 各组薄膜与水的接触角依次为 $7.4^{\circ} 、 101.5^{\circ} 、 114.6^{\circ}$ 、 $119.2^{\circ} 、 129.5^{\circ} 、 131.5^{\circ}$ 。未改性的 $\mathrm{SiO}_{2}$ 薄膜接触角 非常小, 这是因为碱催化制备的 $\mathrm{SiO}_{2}$ 薄膜表面含有 大量的羟基，薄膜易吸附水汽，随着 MTES 的加入， 改性后的 $\mathrm{SiO}_{2}$ 薄膜和水接触角迅速增加到 $101.5^{\circ}$; 随着 MTES 含量进一步增大, $\mathrm{SiO}_{2}$ 薄膜与水的接触 角增长缓慢，当掺杂比增加到 $40 \mathrm{~mol} \%$ 以上时，薄膜 接触角基本上保持不变, 继续增加 MTES 含量对接 触角影响较小。

\subsubsection{MTES 疏水改性机理分析}

碱催化制备的 $\mathrm{SiO}_{2}$ 薄膜由大量的 $\mathrm{SiO}_{2}$ 颗粒堆 积而成, 大量的羟基附着在 $\mathrm{SiO}_{2}$ 颗粒表面, 薄膜即 使在煅烧后还存在大量羟基基团。MTES 的加入实 际上是通过两者中间产物的缩聚反应把- $\mathrm{CH}_{3}$ 引入 到 $\mathrm{SiO}_{2}$ 网络结构中去, 具体的反应过程见反应式 (1)、(2)和(3)。

水解反应:

$$
\mathrm{Si}\left(\mathrm{OC}_{2} \mathrm{H}_{5}\right)_{4}+4 \mathrm{H}_{2} \mathrm{O} \rightarrow \mathrm{Si}(\mathrm{OH})_{4}+4 \mathrm{C}_{2} \mathrm{H}_{5} \mathrm{OH}
$$

$\mathrm{CH}_{3} \mathrm{Si}\left(\mathrm{OC}_{2} \mathrm{H}_{5}\right)_{3}+3 \mathrm{H}_{2} \mathrm{O} \rightarrow \mathrm{CH}_{3} \mathrm{Si}(\mathrm{OH})_{3}+3 \mathrm{C}_{2} \mathrm{H}_{5} \mathrm{OH}$

聚合反应:

$\mathrm{Si}(\mathrm{OH})_{4}+\mathrm{CH}_{3} \mathrm{Si}(\mathrm{OH})_{3} \rightarrow \mathrm{CH}_{3} \mathrm{Si}(\mathrm{OH})_{2}-\mathrm{O}-\mathrm{Si}(\mathrm{OH})_{3}+\mathrm{H}_{2} \mathrm{O}$

为了进一步了解薄膜的疏水机理，利用红外光 谱仪测得各组掺杂 MTES 改性薄膜的红外吸收光谱, 如图 8 所示。从图 8 可以看出, 未掺杂时薄膜在 960 和 $3400 \mathrm{~cm}^{-1}$ 波数处分别出现 $\mathrm{Si}-\mathrm{OH}$ 伸缩振动吸收 峰和 $\mathrm{OH}$ 基团反对称伸缩振动吸收峰 ${ }^{[16]}$, 这

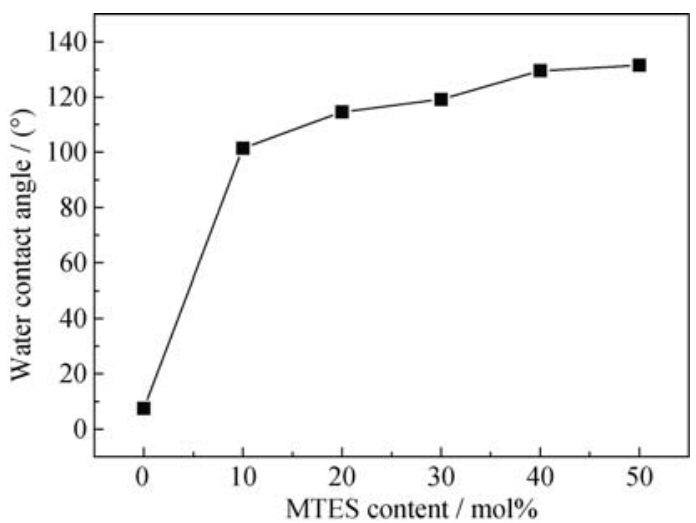

图 7 不同 MTES 掺杂比制备的 $\mathrm{SiO}_{2}$ 薄膜表面接触角的影响 Fig. 7 Surface water contact angles of $\mathrm{SiO}_{2}$ films prepared with different MTES doping ratios 


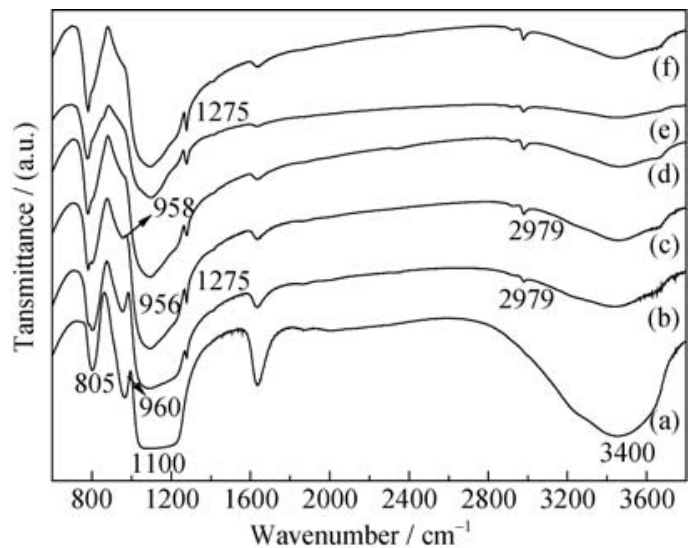

图 8 不同 MTES 掺杂比制备的 $\mathrm{SiO}_{2}$ 薄膜红外光谱

Fig. 8 Infrared spectra of $\mathrm{SiO}_{2}$ films prepared with different MTES doping ratios

(a) 0 ; (b) $10 \mathrm{~mol} \%$; (c) $20 \mathrm{~mol} \%$; (d) $30 \mathrm{~mol} \%$; (e) $40 \mathrm{~mol} \%$; (f) $50 \mathrm{~mol} \%$

就使得空气中的水份极易吸附在薄膜表面, 导致 薄膜透过率下降。各组试样在 $1100 \mathrm{~cm}^{-1}$ 处均有强 的 $\mathrm{Si}-\mathrm{O}-\mathrm{Si}$ 特征吸收峰, 该峰所处位置为 $\mathrm{Si}-\mathrm{O}-\mathrm{Si}$ 伸缩振动峰 ${ }^{[16]}$ 。随着 MTES 的加入, 薄膜的红外光 谱曲线在 $1275 \mathrm{~cm}^{-1}$ 处出现了甲基基团的吸收峰, 该吸收峰是与中心 $\mathrm{Si}$ 原子连接的甲基中碳氢键的 弯曲振动吸收峰 ${ }^{[17]}$ 。随着 MTES 掺杂比例的增加, $1275 \mathrm{~cm}^{-1}$ 处的吸收峰不断增强, 在 $2979 \mathrm{~cm}^{-1}$ 处还 出现了新的吸收峰, 该峰为 $\mathrm{C}-\mathrm{H}$ 键伸缩振动峰, 它随着 MTES 的加入也呈现从无到有, 再不断增强 的变化趋势，这表明由 MTES 和 TEOS 水解中间产 物的共聚合反应制备溶胶中甲基被成功添加到 $\mathrm{SiO}_{2}$ 网络结构中。通过对比掺杂 MTES 溶胶制备的改性 薄膜和未改性薄膜的 IR 谱发现, 在 $960 \mathrm{~cm}^{-1}$ 处的吸 收峰值随着 MTES 掺杂量的增加, 该吸收峰不断 减弱, 在掺杂比为 $30 \%$ 的薄膜中基本上消失了。这 同样说明疏水基团甲基的加入使得薄膜结构中的 亲水基团羟基不断减少，从而使薄膜的疏水性能 不断提高。

\section{4 耐环境性能}

将制备好的宽光谱疏水性减反射膜、宽光谱减 反射膜以及空白玻璃放置于室外两个月后, 取回用 清水冲洗后, 分别测量初始样品与清洗后样品透过 率变化曲线, 如图 9 所示。从图 9 可以看出, 镀有三 层疏水膜的样品放置室外 2 个月后，薄膜平均透过 率相对未处理的三层膜仅下降 $1.61 \%$; 玻璃基底放 置室外 2 个月后, 平均透过率相对未处理的下降了 $4.17 \%$ 。另外, 耐候测试后的疏水薄膜经清洗后透过 率仅下降 0.143\%(400 800 nm), 同样说明薄膜具有 较好的耐候性能。

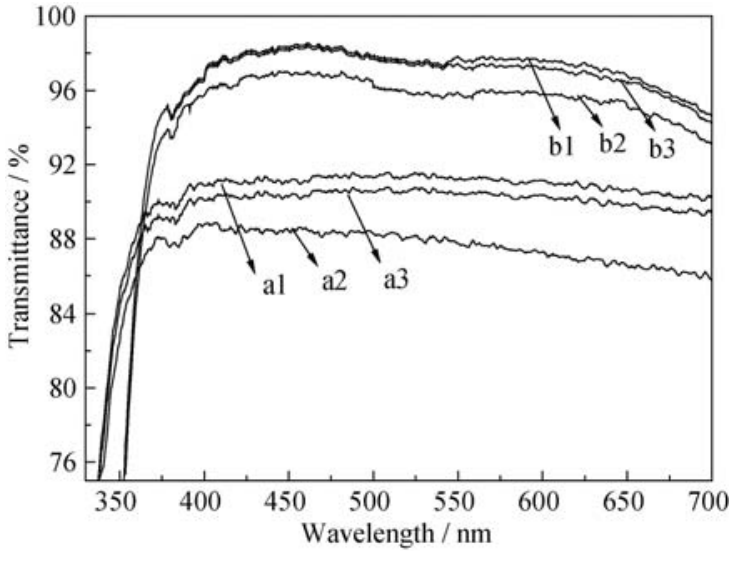

图 9 超白玻璃与三层疏水膜耐候性测试前后透射率曲线

Fig. 9 Transmittances of low-ion glass and triple-layer antireflective films after environment resistant test

$a_{1}$ ) glass, $a_{2}$ ) glass without cleaning, $a_{3}$ ) glass after cleaning, $b_{1}$ ) triple-layer film, $b_{2}$ ) triple-layer film without cleaning, $b_{3}$ ) triple-layer film after cleaning

\section{4 结论}

利用 TFCcal 膜系设计软件，结合溶胶一凝胶技 术探究了宽波段高透过率 $\mathrm{SiO}_{2} / \mathrm{TiO}_{2} / \mathrm{SiO}_{2}-\mathrm{TiO}_{2}$ 减反 膜的制备工艺, 并对薄膜的耐环境性能进行了测试 分析。主要结论如下:

1) 结合 TFCcal 软件膜系设计, 高增透型三层 宽光谱减反膜的膜层、厚度和折射率从内到外依次 为: 内层 $\mathrm{SiO}_{2}-\mathrm{TiO}_{2}, 80.9 \mathrm{~nm}$, 折射率 1.70; 中间层 $\mathrm{TiO}_{2}, 125.0 \mathrm{~nm}$, 折射率 2.2; 外层 $\mathrm{SiO}_{2}, 95.5 \mathrm{~nm}$, 折 射率 1.44 。薄膜在 300 700 $\mathrm{nm}$ 可见光范围内平均 透过率高达 $98 \%$ 左右。

2）利用甲基三乙氧基硅烷(MTES)疏水改性碱 催化制备的 $\mathrm{SiO}_{2}$ 溶胶, 镀膜热处理得疏水 $\mathrm{SiO}_{2}$ 层, 当 MTES 溶胶掺杂比为 $50 \mathrm{~mol} \%$ 时，薄膜与水接触 角为 $131.5^{\circ}$, 薄膜折射率为 1.436 , 基本符合 TFCcal 设计中 $\mathrm{SiO}_{2}$ 层所需折射率值, 在保证薄膜机械强度 的前提下具有优良的疏水改性作用。

3) $\mathrm{SiO}_{2}-\mathrm{TiO}_{2}$ 层、 $\mathrm{TiO}_{2}$ 层和 $\mathrm{SiO}_{2}$ 层分别采用提 拉速度为 190、420 和 $190 \mathrm{~mm} / \mathrm{min}$ 进行制备时, 三 层复合减反膜在 400 700 nm 范围内平均透过率为 $97.03 \%$, 接近理论设计值，且薄膜具有优良的耐环 境性能。

\section{参考文献:}

[1] DEUBENER J, HELSCH G, MOISEEV A, et al. Glasses for solar energy conversion systems. J. Eur. Ceram. Soc. 2009, 29: 1203-1210.

[2] YE H P, ZHANG X X, ZHANG Y L, et al. Preparation of antire- 
flective coatings with high transmittance and enhanced abrasionresistance by a base/acid two-step catalyzed Sol-Gel process. Solar Energy Mater. Solar Cells, 2011, 95(8): 2347-2351.

[3] VICENTE G S, BAYON R, GERMAN N, et al. Long-term durability of Sol-Gel porous coatings for solar glass covers. Thin Solid Films, 2009, 517(10): 3157-3160.

[4] HELSCH G, MÖS A, DEUBENER J, et al. Thermal resistance of nanoporous antireflective coatings on silica glass for solar tower receivers. Solar Energy Mater. Solar Cells, 2010, 94(12): 2191-2196.

[5] PRADO R, BEOBIDE G, MARCAIDE A, et al. Development of multifunctional Sol-Gel coatings: anti-reflection coatings with enhanced self-cleaning capacity. Solar Energy Mater. Solar Cells, 2010, 94(6): 1081-1088.

[6] WANG JIAN-WU, BAI YU-CHEN, YAO WEI, et al. Preparation and investigation of $\mathrm{SiO}_{2} / \mathrm{TiO}_{2}$ antireflective coatings with self-cleaning and scratch-resistant properties. Journal of Inorganic Materials, 2011, 26(7): 769-773.

[7] YAN L H, CHI F T, JIANG X B, et al. Preparation of hydro-oleophobic silica antireflective coating. Journal of Inorganic Materials, 2007, 22(6): 1247-1250.

[8] LI X, SHEN J. A Scratch-resistant and hydrophobic broadband antireflective coating by Sol-Gel method. Thin Solid Films, 2011, 519(19): $6236-6240$.

[9] XIAO B, ZHANG Y L, ZHANG X X, et al. Focus on moisture-resistance and hydrophobicity of $\mathrm{SiO}_{2}$ antireflective film improved by poly(isopropylene oxide) glycerolether. J. Sol-Gel Sci.
Technol., 2011, 60(1): 11-16.

[10] LEI M A, LI F S, SAKAE T, et al. Cost-effective nanoporous $\mathrm{SiO}_{2}-\mathrm{TiO}_{2}$ coatings on glass substrates with antireflective and self-cleaning properties. Appl. Energy, 2013, 112: 1198-1205.

[11] YE L, ZHANG Y, ZHANG X, et al. Sol-Gel preparation of $\mathrm{SiO}_{2} / \mathrm{TiO}_{2} / \mathrm{SiO}_{2}-\mathrm{TiO}_{2}$ broadband antireflective coating for solar cell cover glass. Solar Energy Materials and Solar Cells, 2013, 111: $160-164$.

[12] 唐晋发, 顾培夫, 刘旭等著. 现代光学薄膜技术. 杭州: 浙江大 学出版社, 2006.

[13] NOSTELL P, ROOSA A, KARLSSON B. Optical and mechanical properties of Sol-Gel antireflective films for solar energy applications. Thin Solid Films. 1999, 351: 170-175.

[14] CHANG Y C, MEI G H, CHANG T W, et al. Design and fabrication of a nanostructured surface combining antireflective and enhanced-hydrophobic effects. Nanotechnology, 2007, 18(28): 285 303.

[15] BARTHLOTT W, NEINHUIS C. Purity of the sacred lotus, or escape from contamination in biological surfaces. Planta, 1997, 202(1): $1-8$.

[16] ZHANG X X, CAO C R, XIAO B, et al. Preparation and characterization of polyvinyl butyral/silica hybrid antire flective coating: effect of PVB on moisture-resistance and hydrophobicitye. Sol-Gel Sci. Technol, 2010, 53(1): 79-84.

[17] WADA M, KAMIYA K, NASU H. X-ray diffraction analysis of $\mathrm{SiO}_{2}$ gel prepared from monomethyl-tri-ethoxysilane by the Sol-Gel method. Phys. Chem. Glasses, 2002, 33(2): 56-60. 